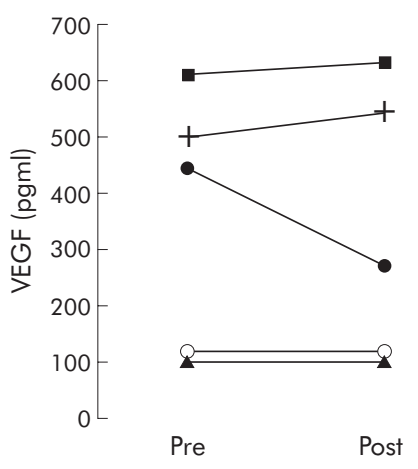

Figure 1 Effect of rofecoxib on serum levels of vascular endothelial growth factor (VEGF) (A) and endostatin (B), measured by enzyme linked immunoassay (R\&D Systems, Abingdon, UK). Lines join paired data from individual patients before (pre) and after (post) rofecoxib therapy $(n=5)$. Symbols for each set of paired data correspond to the same metastases in (A) and (B).

Correspondence to: Professor M A Hull, Molecular Gastroenterology, Leeds Institute of Molecular Medicine, University of Leeds, St James's University Hospital, Leeds LS9 7TF, UK; M.A.Hull@leeds.ac.uk

\section{doi: 10.1136/gut.2005.090571}

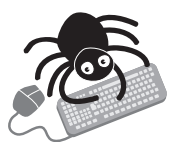

Conflict of interest: declared (the declaration can be viewed on the Gut website at http://www.gutjnl.com/ supplemental).

\section{References}

1 Fenwick SW, Toogood GJ, Lodge JP, et al. The effect of the selective cyclooxygenase-2 inhibitor rofecoxib on human colorectal cancer liver metastases. Gastroenterology 2003; 125:716-29.

2 Ma L, del Soldato P, Wallace JL. Divergent effects of new cyclooxygenase inhibitors on gastric ulcer healing: Shifting the angiogenic balance. Proc Natl Acad Sci U S A 2002:99:13243-7.

3 Leach MO, Brindle KM, Evelhoch JL, et al. Assessment of antiangiogenic and antivascular therapeutics using MRI: recommendations for appropriate methodology for clinical trials. Br J Radiol 2003;76(suppl 1):S87-91.

4 Tofts PS, Brix G, Buckley DL, et al. Estimating kinetic parameters from dynamic contrastenhanced T(1)-weighted MRI of a diffusable tracer: standardized quantities and symbols. J Magn Reson Imaging 1999;10:223-32.

5 Morgan B, Thomas AL, Drevs J, et al. Dynamic contrast-enhanced magnetic resonance imaging as a biomarker for the pharmacological response of PTK787/ZK222584, an inhibitor of the vascular endothelial growth factor receptor tyrosine kinases, in patients with advanced colorectal cancer and liver metastases: Results from two phase I studies. J Clin Oncol 2003;21:3955-64.

6 Van Laarhoven HWM, Rijpkema M, Punt CJA, ef al. Method for quantitation of dynamic MRI contrast agent uptake in colorectal liver metastases. J Magn Reson Imaging 2003; 18:315-20

7 Jackson A, Haroon $\mathrm{H}$, Zhu XP, et al. Breath-hold perfusion and permeability mapping of hepatic malignancies using magnetic resonance imaging and a first-pass leakage profile model. NMR Biomed 2002;15:164-73.

8 Medved M, Karczmar G, Yang C, et al. Semiquantitative analysis of dynamic contrast enhanced MRI in cancer patients: Variability and changes in tumor tissue over time. J Magn Reson Imaging 2004;20:122-8.

9 Jain RK. Normalization of tumor vasculature: An emerging concept in antiangiogenic therapy. Science 2005;307:58-62.

10 Sund M, Hamano $Y$, Sugimoto $H$, et al. Function of endogenous inhibitors of angiogenesis as endothelium-specific tumor suppressors. Proc $\mathrm{Nat}$ Acad Sci U S A 2005; 102:2934-9.

\section{Ulcerative colitis during CTLA-4Ig therapy in a patient with rheumatoid arthritis}

The coexistence of rheumatoid arthritis (RA) and ulcerative colitis (UC) is exceptional, with only a few cases reported in the literature. ${ }^{1-4}$ However, the current use of biological agents can modify the balance of inflammatory mediators and the lymphocytic profiles of the original disease, promoting the emergence of additional conditions. This seems to be the case for a patient with severe RA who developed UC while being treated with CTLA-4Ig (abatacept).

We report the case of a 55 year old male with a seven year history of seropositive RA refractory to treatment with $20 \mathrm{mg} /$ week methotrexate (MTX), $1.5 \mathrm{~g} /$ day sulphasalazine (SSZ), $400 \mathrm{mg} /$ day hydroxychloroquine, and $100 \mathrm{mg} /$ day azathioprine. The patient was included in an CTLA-4Ig $(10 \mathrm{mg} / \mathrm{kg}$ intravenously monthly) plus MTX clinical trial. All other disease modifying antirheumatic drugs were discontinued. During treatment, major clinical and serological responses were achieved (table 1). Fifteen months later, the patient developed diarrhoea, rectal bleeding, crampy abdominal pain, tenesmus, and weight loss. CTLA-4Ig and MTX were discontinued. Stool search for bacteria, ova, and parasites yielded negative results; serological tests for human immunodeficiency virus, hepatitis $\mathrm{B}$, and hepatitis $\mathrm{C}$ were non-reactive. Colonoscopy revealed extensive ulcerative lesions in the sigmoid colon and rectum. Histology showed a dense lymphoplasmocytic inflammatory infiltration distorting the architecture of the mucosa; cryptitis with irregular basal branching and intraluminal abscesses were also apparent and Paneth cells were
B

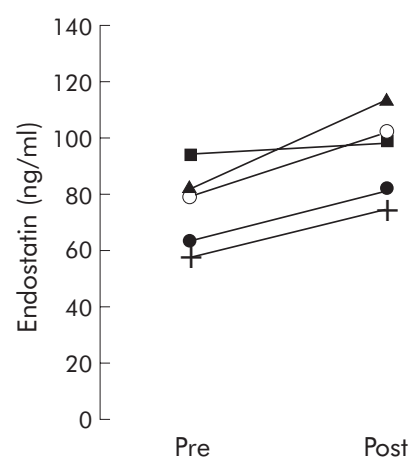

identified in crypt bases. A diagnosis of UC was made and oral mesalamine therapy was started at a dose of $1.6 \mathrm{~g} /$ day with a satisfactory response. The dose of mesalamine was gradually reduced over the next three months until discontinuation.

Four months after CTLA4-Ig withdrawal, the patient remained asymptomatic for UC and the search for blood in stool was negative. However, arthritis was reactivated and a step on regimen with $25 \mathrm{mg} /$ week MTX, $3 \mathrm{~g} /$ day SSZ, $5 \mathrm{mg} /$ day prednisone, and $20 \mathrm{mg} /$ day leflunomide was initiated.

Diverse methods have been proposed to assess the likelihood of a causal connection between environmental exposure (including therapeutic drugs) and an undesirable adverse event. ${ }^{5}$ According to Miller and colleagues, ${ }^{6}$ a series of five primary attribution elements or pertinent questions should be addressed when suspected exposure may be related to a clinical outcome for a given patient: (1) temporal association; (2) lack of likely alternative explanations; (3) dechallenge (improvement in symptoms following discontinuation of the drug); (4) rechallenge; and (5) biological plausibility.

In our case, both temporal association and dechallenge suggest that CTLA-4Ig could be related to the development of UC and autoantibody induction. Analysing other likely alternative explanations, the major infectious causes of bloody diarrhoea were excluded, and no evidence of vasculitis of the mesenteric arteries or amyloidosis was found. Rechallenge was not available because CTLA-4Ig was not reinitiated.

Biological plausibility is also possible considering the following facts: CTLA-4Ig was designed and has been used to block the interactions between CD80/CD86 on antigen presenting cells and CD28 on T lymphocytes, preventing their activation. ${ }^{7}$ However, this same mechanism could alter both the development and maintenance of regulatory $\mathrm{T}$ cells, ${ }^{8}$ which control intestinal inflammation. Also, absence of the cytoplasmic domain of CTLA-4 and hence of the intracellular signal it mediates favours a Th2 biased phenotype. ${ }^{9}$ This could explain the emergence of UC and the presence of autoantibodies, both phenomena related to a Th2 response. ${ }^{10}$

Although four of Miller's primary attribution elements are fulfilled, we cannot prove causality in this single case, especially in the absence of rechallenge. Nevertheless, theoretically, CTLA-4Ig could favour the emergence of Th2 mediated phenomena, an issue that needs to be elucidated.

L M Amezcua-Guerra Department of Immunology, Instituto Nacional de Cardiología Ignacio Chávez, Mexico City, México

Table 1 Clinical and laboratory findings

\begin{tabular}{llllll}
\hline \multicolumn{7}{l}{ CTLA-4lg therapy } \\
\cline { 2 - 6 } Measurement & Baseline & $\mathbf{1 2}$ months & $\mathbf{1 5}$ months & $\mathbf{1 9}$ months & $\mathbf{2 6}$ months \\
\hline Tender joint count & $22 / 28$ & $9 / 28$ & $11 / 28$ & $16 / 28$ & $28 / 28$ \\
Swollen joint count & $18 / 28$ & $2 / 28$ & $8 / 28$ & $12 / 28$ & $24 / 28$ \\
C reactive protein (mg/l) & 22.7 & 7.3 & 5.6 & 42.7 & 50.2 \\
DAS-28 & 6.58 & 4.25 & 4.77 & 6.16 & 7.47 \\
Antinuclear antibodies* & 0 & $1: 40$ & $1: 40$ & 0 & 0 \\
Anti-dsDNA (UI/ml)t & 42 & 13 & 107 & 45 & 30 \\
\hline
\end{tabular}

*Antinuclear antibodies are expressed in dilutions. Indirect immunofluorescence on HEp-2 slides. $\dagger$ Anti-dsDNA, anti-double stranded DNA antibodies (reference range $\leqslant 99 \mathrm{UI} / \mathrm{ml}$ ). 
B Hernández-Martínez, C Pineda

Department of Rheumatology, Instituto Nacional de Cardiología Ignacio Chávez, Mexico City, México

R Bojalil

Department of Immunology, Instituto Nacional de Cardiología lgnacio Chávez, Mexico City, México

Correspondence to: Dr R Bojalil, Department of Immunology, Instituto Nacional de Cardiología lgnacio Chávez, Juan Badiano 1, Sección XVI, Tlalpan 14080, Mexico City, México; bojraf@yahoo.com doi: 10.1136/gut.2006.095539

Conflict of interest: None declared.

\section{References}

Sugisaki K, Honma $\mathrm{F}$, Iwadate $\mathrm{H}$, et al. Ulcerative colitis occurring in the course of rheumatoid arthritis: a case successfully treated with mesalamine enema. Intern Med 2004;43:1046-50.

2 Adachi $Y$, Hinoda $Y$, Takahashi $\mathrm{H}$, et al. Rheumatoid arthritis associated with ulcerative colitis. J Gastroenterol 1996;31:590-5.

3 Boyer F, Fontanges E, Miossec P. Rheumatoid arthritis associated with ulcerative colitis: a case with severe flare of both diseases after delivery. Ann Rheum Dis 2001;60:901.

4 Aydin Y, Ozcakar L, Yildiz M, et al. Liaison between rheumatoid arthritis and ulcerative colitis. Rheumatol Int 2003;23:47-8.

5 Stephens MD. The diagnosis of adverse medical events associated with drug treatment. Adverse Drug React Acute Poisoning Rev 1987;6: 1-35.

6 Miller FW, Hess EV, Clauw DJ, et al. Approaches for identifying and defining environmentally associated rheumatic disorders. Arthritis Rheum 2000;43:243-9.

7 Kremer JM, Westhovens R, Leon M, et al. Treatment of rheumatoid arthritis by selective inhibition of T-cell activation with fusion protein CTLA4lg. N Engl J Med 2003;349:1907-15

8 Greenwald RJ, Freeman GJ, Sharpe AH. The B7 family Revisited. Annu Rev Immunol 2005;23:515-48.

9 Masteller EL, Chuang E, Mullen AC, et al. Structural analysis of CTLA-4 function in vivo. J Immunol 2000; 164:5319-27.

10 Heller F, Florian P, Bojarski C, et al. Interleukin-13 is the key effector Th2 cytokine in ulcerative colitis that affects epithelial tight junctions, apoptosis, and cell restitution. Gastroenterology $2005 ; 129: 550-64$

\section{An unusual case of severe iron deficiency anaemia}

Abnormal iron absorption is a well recognised feature of subtle non-bleeding gastrointestinal conditions, such as coeliac disease or atrophic body gastritis, without otherwise gastrointestinal symptoms. We recently observed a patient with severe iron deficient anaemia mimicking impaired iron absorption in the absence of gastrointestinal pathology.

A 47 year old women was admitted to hospital in 2005 because a haemoglobin level of $6.4 \mathrm{~g} / \mathrm{dl}$ had attracted attention when she was donating blood. She complained of fatigue and occasionally frontal headaches. On physical examination she appeared to have a normal nutritional condition but her skin was pale and she had brittle nails. Despite the severity of the anaemia she appeared well. Pulse frequency and blood pressure were normal. Blood smear showed a strikingly microcytotic hypochromic red blood cell morphology (mean corpuscular volume $56 \mathrm{fl}$, mean corpuscular haemoglobin 15.5 pg/cell, mean cell haemoglobin concentration $27.5 \mathrm{~g} /$ dl) (fig 1). According to this, serum iron was below $5 \mu \mathrm{g} / \mathrm{l}$, serum transferrin was elevated to $3.97 \mathrm{~g} / \mathrm{l}$ and less than $10 \%$ saturated, and serum ferritin concentration was $3 \mathrm{mg} / \mathrm{l}$ Erythrocyte count was normal $\left(4.14 \times 10^{12} / 1\right)$,

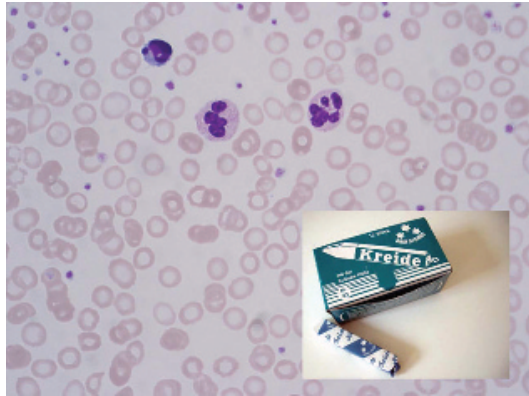

particularly if other causes of abnormal iron absorption have been excluded.

P Schnuelle, T Oberheiden, D Hohenadel U Gottmann, U Benck

Department of Medicine, University Hospita Mannheim, Medical Faculty of the University of Heidelberg, Germany

T Nebe

Institution of Clinical Chemistry, University Hospital Mannheim, Medical Faculty of the University of Heidelberg, Germany

H J Krammer, F J van der Woude, R Birck Department of Medicine, University Hospital Mannheim, Medical Faculty of the University of Heidelberg, Germany

Figure 1 Red cell smear at the time of admission, with typical features of severe iron deficiency. Erythrocytes are smaller in size (microcytosis) and show expansion of their central area of pallor, thereby appearing to have only a thin rim of haemoglobin (hypochromia). Insert: type of purchasable crayon ingested. Each pack contains 12 pieces.

indicating enduring iron deficient erythropoiesis rather than bleeding. In fact, the guaiac smear test was negative and endoscopies of the gastrointestinal tract revealed no abnormalities. Gynaecological examination excluded uterine bleeding. Exploring her food patterns she confessed to regular eating of crayons for more than nine years to compensate for a sulky taste. She had such a craving for clean white crayons that she had increased her intake to 20 packages per month (fig 1). Three days after cessation, oral iron absorption tests were normal. Starting treatment with an oral iron preparation resulted in reticulocytosis with a peak of $7.8 \%$ after 10 days.

This exceptional case of severe iron deficiency anaemia related to long term ingestion of large amounts of purchasable crayons clearly meets the diagnostic criteria of adult pica. Pica comes from the Latin word for magpie, a bird known for its large and indiscriminate appetite, and implies a qualitative eating disorder defined by oral ingestion of non-food items for at least one month. ${ }^{1}$ Pica is most common in those with developmental disabilities, including autism and mental retardation, and in children. Apart from sporadic cases due to a specific underlying psychiatric disorder, pica also features a culture bound syndrome worldwide. Iron deficiency is one peculiar finding quite commonly associated with pica. Anaemia, dwarfism, and hypogonadism have been related to clay eating among children and women from rural areas in Turkey and Iran. ${ }^{2}$ Eating of chalk among children and Black pregnant and nonpregnant women in middle Georgia reflects another example of a socioculturally transmitted form of pica without other apparent psychopathology. ${ }^{3}$ The basis of the bizarre behaviour in pica still remains obscure Specific nutritional deficiencies of particular trace elements may trigger the unusual cravings for non-food items. ${ }^{4}$ Some of these materials bind iron in the gastrointestinal tract, worsening the deficiency. Pica has recently been identified as a predominant risk factor for anaemia in pregnant women in a large case control study from the Sudan. ${ }^{5}$ The type of crayon ingested by our patient consisted of pure calcium sulphate. It is conceivable that the bioavailability of dietary iron was markedly impaired in the presence of abundant calcium sulphate.

Albeit a rare condition in Central Europe, adult pica should be considered for differential diagnosis of chronic iron deficiency anaemia,
Correspondence to: Dr P Schnuelle, University Hospital Mannheim, Vth Medical Clinic, TheodorKutzer Ufer 1-3, 68135 Mannheim, Germany peter.schnuelle@med5.ma.uni-heidelberg.de doi: $10.1136 /$ gut.2006.094680

Conflict of interest: None declared.

\section{References}

1 Diagnostic and Statistical Manual of Mental Disorders, 4th edn., Washington, DC: American Psychiatric Association 2000 .

2 Arcasoy A, Cavdar AO, Babacan E. Decreased iron and zinc absorption in Turkish children with iron deficiency and geophagia. Acta Haematol 1978:60:76-84

3 Grigsby RK, Thyer BA, Waller RJ, et al. Chalk eating in middle Georgia: a culture-bound syndrome of pica? South Med J 1999;92:190-2.

4 Diamond JM. Evolutionary biology. Dirty eating for healthy living. Nature 1999;400:120-1.

5 Adam I, Khamis AH, Elbashir MI. Prevalence and risk factors for anemia in pregnant women of eastern Sudan. Trans R Soc Trop Med Hyg 2005:99:739-43.

\section{NOTICE}

\section{Second ESPGHAN Capri Meeting. Genetic factors in functional and structural GI disorders: scientific advances and clinical implications}

This meeting will be held on 12-15 October 2006 at the Grand Hotel Quisisana, Capri, Naples, Italy. The scientific programme highlights new insights into the contribution of genetic factors to a wide range of GI diseases, including the functional GI disorders (FGID). Speakers are instructed to translate scientific advances into meaningful information for the clinician. The meeting is expected to promote 1 ) further research into disorders wherein genetic aspects have a recognised or newly appreciated impact, as well as 2) improved management of patients with these disorders. The discussed scientific advances may have broad relevance, extending to GI diseases in which genetic factors remain poorly recognised. Susceptibility factors for FGID (host and environment) will be revealed along with their effects on clinical management. For more information please contact: Professor Annamaria Staiano or Riccardo Troncone, Department of Pediatrics, University of Naples "Federico II", Via S. Pansini, 5-80131 Naples; tel: +39 0817462679 ext 3383; fax: +39 0815469811; email: staiano@unina.it or troncone@unina.it; website: http://www.messaggi-events.org/. 